\title{
Las trayectorias profesionales de los estudiantes de máster en Educación: un análisis desde las expectativas y la transición
}

\author{
María Delia Justiniano Domínguez \\ Universidad Católica Boliviana. Bolivia. \\ mjustiniano@ucbscz.com.bo
}

Recibido: 3/4/2018

Aceptado: 12/4/2018

Publicado: 15/7/2019

\section{Resumen}

El artículo describe la realidad de los estudios postgraduales en Educación en el contexto boliviano. Los cambios estructurales de la educación superior boliviana exigen el análisis de la formación desde dos puntos de vista: el sociocognitivo y el estructural. A partir del fundamento que permiten establecer las teorías estructuralistas de Lent, Cabrera, Tinto, Pascarella y Terenzini, entre otros, se presenta un postulado centrado en el análisis sobre las trayectorias universitarias, los itinerarios de la formación postgradual, las teorías sobre la transición y las expectativas de los alumnos. El método utilizado en la investigación es de carácter cuantitativo y sirve para definir las características del instrumento de recolección de datos y el procedimiento para su aplicación. Los resultados permiten visualizar elementos clave como la procedencia académica, la elección de los estudios, el proyecto profesional y el cumplimiento de objetivos que permiten estructurar procesos de trayectoria universitaria.

Palabras clave: expectativa; transición; universidad; motivación para los estudios

Resum. Les trajectòries professionals dels estudiants de màster en Educació: una anàlisi des de les expectatives $i$ la transició

L'article descriu la realitat dels estudis de postgrau en el context bolivià, específicament se centra en els màsters en Educació que s'imparteixen a la ciutat de Santa Cruz (Bolívia). Els canvis estructurals de l'educació superior boliviana exigeixen que la formació sigui analitzada des de dos punts de vista: el sociocognitiu i l'estructural. A partir del fonament que permeten establir les teories estructuralistes de Lent, Cabrera, Tinto, Pascarella i Terenzini, entre d'altres, es presenta un postulat centrat en l'anàlisi sobre les trajectòries universitàries, els itineraris de la formació postgradual, les teories sobre la transició i les expectatives dels alumnes. El mètode utilitzat en la recerca és de caràcter quantitatiu i serveix per definir les característiques de l'instrument de recol-lecció de dades i el procediment per aplicar-les. Els resultats permeten visualitzar elements clau com ara la procedència acadèmica, l'elecció d'estudis, el projecte professional i el compliment d'objectius que permeten estructurar processos de trajectòries universitàries.

Paraules clau: expectativa; transició; universitat; motivació per als estudis 


\begin{abstract}
Professional trajectories of master's students in education: An analysis of expectations and transition
\end{abstract}

This article describes the reality of master's programs in education in Santa Cruz, Bolivia. Current changes in Bolivian higher education are analyzed from a socio-cognitive and structural approach. Based on the works of Lent, Cabrera, Tinto Pascarella and Terenzini, university trajectories, postgraduate educational itineraries, and transition and expectancy theories are analyzed. A quantitative method was used to define the features of the data collection instrument and process. The results reveal that academic background, choice of degree and the achievement of objectives are key in structuring postgraduate trajectory processes.

Keywords: expectations; transition; university; academic motivation

\title{
Sumario
}

1. Introducción 6. Proyecto profesional y cumplimiento

2. La transición de los másteres de expectativas

en educación

3. Factores inmersos en el estudio de las trayectorias universitarias

7. Las transiciones académicas según tipologías de acceso

4. Método

8. Abandono de los estudios de máster

5. Resultados

9. Conclusiones

Referencias bibliográficas

\section{Introducción}

La educación superior en Bolivia atraviesa un proceso de transformación, no solo marcado por la ley educativa 070 , que inició su ejecución el año 2008, sino también por aspectos estructurales relacionados con el crecimiento de la población y de las universidades privadas, así como con el incremento y la diversidad de los grados y de los postgrados ofrecidos.

En la década de 1990 existían trece ofertas postgraduales en todo el país y en la actualidad se registran más de mil programas de dicho nivel académico en diferentes sectores (CEUB, 2015a). La oferta se ha potenciado por la normativa del sistema educativo en la que se exige a todos los docentes de educación superior que cuenten con grado de máster en el caso de las universidades públicas y de diplomatura en las universidades privadas (Ley 070). El escenario de la formación postgradual en Educación configura una complejidad determinada por la diversidad en el diseño formativo entre universidades, las restricciones en las homologaciones entre el sistema fiscal (público) y privado y el alto nivel de deserción y, en consecuencia, los bajos niveles de titulación. Según información del CEUB (2015b), en general, solo entre el 2\% y el 5\% logra titularse como máster.

Estos aspectos nos impulsan a analizar la calidad de los postgrados desde los procesos de la transición, las trayectorias y las expectativas de los estudian- 
tes de máster, con el propósito de buscar mecanismos de mejora desde modelos integrales e intervenciones localizadas.

\section{La transición de los másteres en educación}

Desde un análisis sociológico de la transición (Justiniano, 2007; Casals, 1996), las organizaciones educativas deben favorecer la de los estudiantes de máster y llevar adelante cambios que favorezcan este proceso. Así, la gradualidad con que se llevan los cambios estructurales, la dirección y las estrategias que se adoptan en términos de gestión nos permite asumir que los dispositivos institucionales deben articularse entre sí para lograr el cambio estructural.

Resulta importante analizar la transformación planteada en la ley 070 , que exige la transformación de las trayectorias académicas de los estudios de postgrado en términos de créditos, horas académicas y normativas para la aceptación de los postgrados. En la ciudad de Santa Cruz, de las tres universidades pertenecientes al sistema fiscal y de las catorce privadas, solo tres desarrollan el programa de formación de postgrado en Educación Superior (ver tabla 1).

Los alumnos de los másteres realizan su formación con el propósito, entre otros, de desarrollar competencias que les permitan una mejor práctica o la inserción al sistema educativo superior. Siguiendo a Lazarus y Folkman (1996), la transición está condicionada por el cambio de estructuras cognitivas y afectivas. Este cambio se expresa en el desarrollo de competencias, por tanto, la percepción sobre el mencionado desarrollo es relevante. El análisis de la biografía personal o de la trayectoria profesional de los postulantes al concluir la formación de máster nos muestra que, en muchos casos, se trata de un colectivo de profesionales que están continuando su formación y que cuentan con una trayectoria profesional en el área de la docencia o que tienen intenciones de ejercerla.

Tabla 1. Relación entre universidad, oferta educativa y número de estudiantes (gestión 2013)

\begin{tabular}{|c|c|c|c|c|c|c|}
\hline Universidades & Categoría & Postgrados en educación & 1 & 2 & 3 & 4 \\
\hline $\begin{array}{l}\text { Autónoma } \\
\text { Gabriel René } \\
\text { Moreno }\end{array}$ & Pública & $\begin{array}{l}\text { Diplomado, especialidad y maestría } \\
\text { en Educación Superior }\end{array}$ & 350 & 202 & 108 & 22 \\
\hline $\begin{array}{l}\text { Escuela Militar } \\
\text { de Ingeniería }\end{array}$ & Convenio & $\begin{array}{l}\text { Diplomado, especialidad y maestría } \\
\text { en Educación Superior }\end{array}$ & 49 & 45 & 36 & 9 \\
\hline $\begin{array}{l}\text { Nacional del } \\
\text { Oriente }\end{array}$ & Privada & $\begin{array}{l}\text { Diplomado, especialidad y maestría } \\
\text { en Educación Superior con un enfo- } \\
\text { que en Neurociencias } \\
\text { Diplomado en Tecnología Educativa } \\
\text { Diplomado en Docencia }\end{array}$ & 60 & - & - & - \\
\hline $\begin{array}{l}\text { Aquino de } \\
\text { Bolivia }\end{array}$ & Privada & Maestría en Educación Superior & 50 & 29 & 15 & 8 \\
\hline Nur & Privada & Diplomado en Educación Superior & 48 & - & - & 13 \\
\hline Total & & & 207 & 276 & 159 & 52 \\
\hline
\end{tabular}

Fuente: elaboración propia. 


\section{Factores inmersos en el estudio de las trayectorias universitarias}

El debate conceptual y operativo en torno al estudio de las trayectorias universitarias y su relación con los mecanismos de eficiencia de los sistemas educativos ha estado vinculado con la permanencia, la retención y la graduación.

Entre los factores que explican la permanencia destacan los institucionales, que se encuentran relacionados con la integración académica y social, fundamental en la teoría de persistencia del modelo de Tinto (1988), y el soporte familiar, determinante en la capacidad de superar los objetivos académicos y potenciar la autoeficacia (Perna y Titus, 2005). El apoyo institucional es clave para estimular el desarrollo académico (Karp, 2011; Scott-Clayton, 2011), la utilización del tiempo y los hábitos de estudios (Nonis y Hudson, 2010) y la relación entre el profesorado y el estudiante (Lillis, 2012; HEFCE, 2005; Lotkowski, Robbins y Noeth, 2004; Glogowska, Young y Lockyer, 2007). Asimismo, según Yorke y Longden (2008), hay que tener presentes los factores personales, puesto que son determinantes para la permanencia. Por ello, deben tenerse en cuenta las características sociodemográficas (edad, género, etnia, situación laboral y financiera) (Cabrera, Burkum, Lanasa y Bibo, 2016; Crawford y Harris, 2008); el soporte familiar (Figuera y Torrado, 2013); las variables sociocognitivas que explican las trayectorias de continuidad en la formación (Cabrera et al., 2016; Crawford y Harris, 2008), y la experiencia académica previa (centro de estudios, elección de los estudios, rendimiento, hábitos de estudio).

Completando el modelo integrador de Tinto (1988), el enfoque sociocognitivo es pertinente para entender y profundizar en la interacción de los factores sociocognitivos que participan en el proceso de ajuste académico y en la satisfacción del estudiante en la universidad. Dentro de este enfoque, una de las formulaciones más emergentes es el modelo de ajuste psicosocial y satisfacción académica de Lent (2004), utilizado en nuestro estudio como referente teórico. Según el modelo, las expectativas de autoeficacia y el soporte social son dos factores explicativos clave, al actuar directamente sobre los diversos indicadores de integración académica y, a la vez, indirectamente a través de la influencia sobre la variable actividad hacia la meta, precursora de la integración (Lent, Taveira, Sheu y Singley, 2009).

Diversas investigaciones han profundizado en el valor predictivo de los factores personales en las trayectorias de retención. Se confirma que las variables demográficas (edad, género, etnia, situación laboral y financiera) se asocian a trayectorias con un mayor riesgo de abandono (Crawford y Harris, 2008). Igualmente, las variables sociocognitivas se consideran básicas en la explicación de trayectorias de continuidad, como son la autoestima, el estrés o la autoeficacia (Ojeda, Navarro y Flores, 2011). También la experiencia académica previa (centro de estudios, rendimiento, hábitos de estudio, vía de acceso a la universidad, apoyo académico, competencias previas) se muestra relevante, especialmente en el primer año de universidad (Yorke y Longden, 2008), lo que influye en los siguientes años de carrera (Pascarella y Terenzini, 2005). 
El análisis de la influencia de los contextos en que participa el estudiante aporta interesantes hallazgos en la resolución de la transición. El apoyo familiar es una variable significativa en la explicación de la integración académica y social del estudiante. Como aluden Perna y Titus (2005), los alumnos universitarios con una alta percepción de soporte familiar confían más en su capacidad para superar los objetivos académicos, tienen mayores niveles de autoeficacia en sus estudios y son más autónomos académicamente y socialmente. Sin embargo, cuando la familia no puede asegurar los recursos necesarios para sufragar el coste universitario existe riesgo de abandono (Fike y Fike, 2008). Por otro lado, el apoyo institucional es clave para la retención de los estudiantes cuando estimula el desarrollo académico clarificando objetivos y demandas, orienta sobre cómo conocer y adaptarse al contexto universitario y, además, crea espacios para que el estudiante pueda ser un miembro activo de la comunidad universitaria (Karp, 2011; Scott-Clayton, 2011). Además, se identifican también otros factores institucionales que explican la persistencia universitaria, por ejemplo: las características de las enseñanzas de la institución, las ayudas pedagógicas y de orientación, la demanda de trabajo exigido, la calidad del profesorado o el clima en las aulas (Tinto, 1988). Referente a la actividad académica, es significativa la relación con la utilización del tiempo y los hábitos de estudio (Nonis y Hudson, 2010), así como la relación entre el profesorado y el estudiante (Lillis, 2012). Finalmente, respecto al soporte social de los compañeros y de los amigos, así como las acciones institucionales para fomentar y facilitar el establecimiento de relaciones sociales entre los estudiantes al inicio de su vida en la universidad, se señala que contribuyen de manera significativa a incrementar los recursos personales de afrontamiento de la transición (Karp, 2011; Strydom y Mentz, 2010).

El papel de las expectativas, entendidas como la fuerza de una tendencia a actuar en determinada forma (Vroom, 1964), llevará a explicar que la elección de un tipo de estudios postgraduales por parte de los profesionales está mediada por el nivel de logros que se espera. Siguiendo a Intxausti, Etxeberria y Joaristi (2014) y Stull (2013), la expectativa se refiere a la esperanza de alcanzar cierto logro en la medida en que se proporcionen las oportunidades deseadas para ello, o la creencia de lo que probablemente ocurrirá en el futuro. En relación con las expectativas de formación postgradual, estas se referirán al nivel competencial que se quiere alcanzar y a la satisfacción respecto a las mismas.

Se ha evidenciado una relación positiva entre las expectativas sobre la educación y el rendimiento académico (Galindo y Sheldon, 2012; Cox, Jament y Tarry, 2011; Rivera y Milicic, 2006), como también una asociación entre el nivel socioeconómico y el interés por los estudios. Las personas de condiciones socioeconómicas más bajas desvalorizan los estudios, puesto que consideran que se trata de una pérdida de tiempo que escasamente influirá en el futuro laboral (Weinberg, 2009) y, en sentido inverso, aquellas personas de altos ingresos económicos esperan alcanzar los mayores niveles educativos.

Este trabajo trata de valorar las trayectorias formativas y laborales, así como el cumplimiento de las expectativas y de los factores facilitadores o inhibidores 
de la transición académica de los estudiantes de másteres en Educación, para lo que se pretende:

- Analizar las procedencias académicas y la elección de los estudios según la tipología de acceso de los universitarios de máster en Educación.

- Conocer el proyecto profesional y el cumplimiento de las expectativas como factores facilitadores de la transición académica.

- Analizar los factores facilitadores o inhibidores de la transición académica.

\section{Método}

La investigación fue abordada desde un enfoque cuantitativo, se trata de un estudio descriptivo, de carácter exploratorio a través de un cuestionario (versión papel) dirigido a estudiantes de másteres en Educación de las universidades Gabriel René Moreno (UGRM), Escuela Militar de Ingeniería (EMI) y Universidad de Aquino de Bolivia (UDABOL).

\subsection{Población y muestra}

La participación ha sido de 88 estudiantes pertenecientes a 3 universidades de la ciudad de Santa Cruz de la Sierra. El criterio principal para definir los diferentes centros participantes fue su tipología (pública y privada), la existencia de una trayectoria formativa en máster en Educación y la accesibilidad a los datos sobre los estudiantes (ver la tabla 2).

Al tratarse de una población reducida, se han solicitado datos del 100\% de la población. La muestra es intencional, considerando la accesibilidad y la predisposición para responder a las encuestas.

Tabla 2. Muestra participante de estudiantes

\begin{tabular}{lcc}
\hline Universidad & $\mathbf{N}^{{ }^{\circ}}$ total & Muestra \\
\hline Gabriel René Moreno & 108 & 51 \\
Escuela Militar de Ingeniería & 36 & 23 \\
\hline UDABOL & 22 & 14 \\
\hline Totales & $\mathbf{1 6 6}$ & $\mathbf{8 8}$ \\
\hline
\end{tabular}

Fuente: elaboración propia.

\subsection{Instrumentalización}

Las dimensiones de análisis que fueron utilizadas, con sus subdimensiones y variables respectivas, permitieron delimitar el campo de conocimiento y definir cada uno de los ítems. A partir de esta descripción se elaboró un cuestionario (ver la tabla 3 ). 
Tabla 3. Definición operacional utilizada para el cuestionario

\begin{tabular}{|c|c|c|c|}
\hline Dimensiones & Definición & Categorías & Variables \\
\hline \multirow[b]{2}{*}{$\begin{array}{l}\text { Procedencia } \\
\text { académica }\end{array}$} & \multirow[b]{2}{*}{$\begin{array}{l}\text { Las características } \\
\text { sociodemográficas } \\
\text { y de acceso a los } \\
\text { estudios de másteres }\end{array}$} & $\begin{array}{l}\text { Datos } \\
\text { sociodemográficos }\end{array}$ & $\begin{array}{l}\text { Lugar de nacimiento } \\
\text { Edad } \\
\text { Cambio geográfico } \\
\text { Sexo } \\
\text { Situación familiar } \\
\text { Número de hijos }\end{array}$ \\
\hline & & Acceso al máster & $\begin{array}{l}\text { Este máster fue tu primera } \\
\text { opción } \\
\text { Año de inicio } \\
\text { Año de fin } \\
\text { Matrícula } \\
\text { Financiamiento económico } \\
\text { Formación previa finalizada } \\
\text { Rendimiento académico } \\
\text { previo }\end{array}$ \\
\hline Trayectoria laboral & $\begin{array}{l}\text { El itinerario profesio- } \\
\text { nal antes de ingresar } \\
\text { en los estudios de } \\
\text { másteres }\end{array}$ & Itinerario & $\begin{array}{l}\text { Tipología de acceso al } \\
\text { máster } \\
\text { Situación laboral previa } \\
\text { Situación laboral posterior }\end{array}$ \\
\hline \multirow{3}{*}{$\begin{array}{l}\text { Elección del } \\
\text { máster }\end{array}$} & \multirow{3}{*}{$\begin{array}{l}\text { El valor que da el } \\
\text { postulante a los estu- } \\
\text { dios a los motivos de } \\
\text { elección }\end{array}$} & $\begin{array}{l}\text { Valor profesional } \\
\text { y económico }\end{array}$ & Motivos \\
\hline & & Valor formativo & Motivos \\
\hline & & Valor añadido & Motivos \\
\hline $\begin{array}{l}\text { Tipología de } \\
\text { acceso }\end{array}$ & $\begin{array}{l}\text { Características de los } \\
\text { motivos para ingresar } \\
\text { en el máster }\end{array}$ & Perfil & $\begin{array}{l}\text { Motivo principal para acce- } \\
\text { der al máster }\end{array}$ \\
\hline $\begin{array}{l}\text { Proyecto } \\
\text { profesional }\end{array}$ & $\begin{array}{l}\text { Conjunto de factores } \\
\text { que permiten contar } \\
\text { con una proyección } \\
\text { de la carrera }\end{array}$ & Orientación & Situación laboral \\
\hline \multirow{2}{*}{$\begin{array}{l}\text { Satisfacción } \\
\text { académica }\end{array}$} & \multirow{2}{*}{$\begin{array}{l}\text { Factores que inciden } \\
\text { en el cumplimiento de } \\
\text { expectativas en rela- } \\
\text { ción con la formación }\end{array}$} & $\begin{array}{l}\text { Con la experiencia } \\
\text { formativa }\end{array}$ & Experiencia \\
\hline & & Clima educativo & Experiencia \\
\hline $\begin{array}{l}\text { Factores } \\
\text { favorecedores } \\
\text { o inhibidores } \\
\text { de la transición }\end{array}$ & \multicolumn{2}{|c|}{$\begin{array}{l}\text { Elementos que contribuyen o que dificultan } \\
\text { la integración o el rechazo de los estudios }\end{array}$} & $\begin{array}{l}\text { Has pensado abandonar } \\
\text { Sentimientos con la expe- } \\
\text { riencia del máster }\end{array}$ \\
\hline $\begin{array}{l}\text { Transición acadé- } \\
\text { mico-laboral }\end{array}$ & \multicolumn{2}{|c|}{$\begin{array}{l}\text { El cambio de estructuras del conocimiento } \\
\text { que amplían las posibilidades de inserción } \\
\text { a un trabajo }\end{array}$} & $\begin{array}{l}\text { Utilidad de la formación } \\
\text { Intereses profesionales } \\
\text { Oportunidad laboral }\end{array}$ \\
\hline
\end{tabular}

Fuente: elaboración propia. 


\subsection{Procedimiento}

Para la recogida de información se han establecido diferentes fases en función del trabajo de campo:

- Planificación y diseño del cuestionario. Se procedió a definir operacionalmente las categorías de análisis y se continuó con la construcción del mismo, con respuestas escala Likert (de 1 a 6 ) y preguntas abiertas.

- Validación del cuestionario. Se realizó a partir de jueces externos (5 expertos) que consideraron los criterios de univocidad, coherencia y pertinencia para cada ítem e importancia.

- Revisión y ajuste del cuestionario. Una vez realizada la revisión por parte de los jueces externos, se procedió a la reducción y a la delimitación de ítems. El cuestionario quedó reducido de 40 a 28 ítems.

- Aplicación del cuestionario. De acuerdo con el listado otorgado por las universidades, se procedió a su aplicación a los cursantes que estaban en el último módulo del máster.

- Vaciado de datos en el programa SPSS. Se elaboró una matriz de datos.

- Procedimiento y análisis de datos. Se realizaron análisis estadísticos descriptivos e inferenciales, se sustrajeron las medias, las frecuencias, el ANOVA y las pruebas post oc.

\section{Resultados}

El análisis de los resultados se realizó en función de los objetivos planteados. La trayectoria de los estudiantes de máster, el cumplimiento de sus expectativas y sus procesos de transición fueron examinados desde la procedencia académica, la elección de los estudios, el proyecto profesional, la transición, la satisfacción de los usuarios y el abandono.

\subsection{Procedencia académica y elección de los estudios}

La procedencia académica nos permite conocer las trayectorias profesionales de los encuestados y asociarlos a las características de la elección de los másteres.

Las características sociobiográficas brindan datos básicos sobre la muestra. Esta información permite explicar las posibles diferencias en la trayectoria profesional por grupos.

Sobre la muestra total $(N=88)$ se observa un porcentaje levemente superior de mujeres (el 51\%) en relación con el número de varones (el 49\%). El mayor rango de edad se encuentra entre los 26 y los 30 años (ver la tabla 4). Los estudios postgraduales han implicado un cambio geográfico para el 23\% de los encuestados. El 40\% viven con la familia de origen, el 38\% son independientes con hijos y el 16\% son independientes sin hijos. El 63\% trabaja a tiempo completo, el $26 \%$ lo hace a tiempo parcial y el resto no trabaja. El $42 \%$ son recién titulados, el $38 \%$ son séniores y el $10 \%$, júniores. 
Tabla 4. Rango de edades

\begin{tabular}{ccc}
\hline Rango & $\boldsymbol{N}$ & $\%$ \\
\hline $22-25$ & 7 & 8 \\
$26-30$ & 20 & 30 \\
$31-35$ & 16 & 18 \\
$36-40$ & 19 & 21 \\
$41-45$ & 13 & 14 \\
\hline $46-50$ & 7 & 8 \\
\hline $51-60$ & 5 & 5 \\
\hline
\end{tabular}

Fuente: elaboración propia.

Tabla 5. Motivaciones para elegir los estudios de máster en Educación

\begin{tabular}{llc}
\hline Recién titulados & Especialización profesional & 78,3 \\
$N=37$ & Cambio del área profesional & 15,0 \\
& Promoción laboral & 7,4 \\
& Redefinición profesional & 3,7 \\
\hline Experiencia sénior & Especialización profesional & 54,2 \\
$N=35$ & Cambio de área profesional & 34,2 \\
& Promoción laboral & 11,4 \\
\hline Experiencia júnior & Especialización profesional & 62,5 \\
$N=8$ & Cambio de área profesional & 25,0 \\
& Promoción laboral & 1,2 \\
\hline
\end{tabular}

Fuente: elaboración propia.

La identificación de motivaciones y el grado de importancia para seguir los estudios de másteres inciden en la descripción de los factores internos de las trayectorias profesionales. En el caso de los encuestados se evidencian leves diferencias entre las motivaciones expresadas. Los grupos de júniores y séniores plantean la formación postgradual en educación como una posibilidad de cambio de área profesional y prioritariamente como la búsqueda de una especialización profesional, aunque en menor proporción en relación con los recién titulados. Para este último grupo, la prioridad de su formación postgradual es la especialización profesional (ver la tabla 5).

En la muestra se destaca que entre los 36 y 45 años se obtienen medias más altas que en los mayores de 45 años $(p<0,05)$ en lo que respecta a la especialización profesional. En general, no se encuentran diferencias entre las elecciones que realizan los estudiantes de máster en función del tipo de experiencia.

La mejora económica como motivación es un factor que influye $(p<0,05)$ en los que recién terminaron sus estudios (media $=4,44)$, en comparación con los júniores (media $=3,83$ ). El resto de elecciones no aporta diferencias significativas con relación a las tipologías estudiadas. 


\section{Proyecto profesional y cumplimiento de expectativas}

Los indicadores del proyecto profesional nos permiten conocer las expectativas de autoeficacia y soporte social que favorecen al proyecto profesional (ver la tabla 6).

Tabla 6. Indicadores de proyecto profesional

\begin{tabular}{|c|c|c|c|}
\hline Ítems & Media & Dt & $\mathbf{V z}$ \\
\hline $\begin{array}{l}\text { Me hubiese gustado contar con más ayuda e información antes } \\
\text { de matricularme }\end{array}$ & 3,68 & 1,685 & 2,838 \\
\hline Conozco cuáles son mis habilidades, lo que soy capaz de hacer & 5,25 & 0,810 & 0,656 \\
\hline $\begin{array}{l}\text { Conozco el lugar donde tengo que dirigirme para asesorarme } \\
\text { sobre becas que me interesen y que me ayuden a continuar mis } \\
\text { estudios }\end{array}$ & 2,64 & 1,646 & 2,711 \\
\hline $\begin{array}{l}\text { Soy consciente de lo que me puede ayudar a ir bien en este } \\
\text { máster }\end{array}$ & 4,68 & 1,146 & 1,314 \\
\hline $\begin{array}{l}\text { Tengo claro lo que me interesa de aquí de cara a mi futuro pro- } \\
\text { fesional }\end{array}$ & 5,35 & 0,967 & 0,936 \\
\hline $\begin{array}{l}\text { Conozco cuáles son mis puntos fuertes y mis puntos débiles en } \\
\text { este máster }\end{array}$ & 4,78 & 1,195 & 1,428 \\
\hline $\begin{array}{l}\text { Conozco qué estudios o trabajos puedo realizar cuando finalice } \\
\text { este máster }\end{array}$ & 5,08 & 1,229 & 1,511 \\
\hline $\begin{array}{l}\text { Soy consciente de cuáles son las exigencias de los puestos de } \\
\text { trabajo a los que puedo acceder }\end{array}$ & 4,85 & 1,260 & 1,589 \\
\hline $\begin{array}{l}\text { Tengo conocimientos sobre las diferentes formas de acceder a } \\
\text { un puesto de trabajo }\end{array}$ & 4,31 & 1,408 & 1,983 \\
\hline $\begin{array}{l}\text { Desde que estoy en este máster tengo más claro lo que quiero } \\
\text { hacer con mi vida }\end{array}$ & 4,63 & 1,329 & 1,766 \\
\hline $\begin{array}{l}\text { Soy capaz de solucionar todas las dudas que me surgen sobre } \\
\text { este máster }\end{array}$ & 4,31 & 1,077 & 1,159 \\
\hline Sé valorar para qué puestos de trabajo estoy preparado & 5,00 & 1,017 & 1,035 \\
\hline Tengo el valor de asumir mis errores al tomar cualquier decisión & 5,30 & 0,941 & 0,886 \\
\hline $\begin{array}{l}\text { Sé hacia dónde tengo que dirigirme para encontrar posibles } \\
\text { ofertas de trabajo }\end{array}$ & 4,32 & 1,506 & 2,269 \\
\hline $\begin{array}{l}\text { No me importaría tener puestos de trabajo de mucha responsa- } \\
\text { bilidad }\end{array}$ & 5,01 & 1,340 & 1,795 \\
\hline Me resultó fácil decidir hacer los estudios que estoy realizando & 4,91 & 1,167 & 1,363 \\
\hline $\begin{array}{l}\text { Conozco los pasos a dar para tomar una decisión adecuada res- } \\
\text { pecto a mis estudios o a mi trabajo }\end{array}$ & 4,94 & 0,992 & 0,984 \\
\hline $\begin{array}{l}\text { Conozco los pasos que tengo que dar para montar una empresa } \\
\text { o trabajar como autónomo }\end{array}$ & 4,36 & 1,384 & 1,917 \\
\hline Sé valorar qué puestos de trabajo me interesan más & 5,11 & 1,006 & 1,012 \\
\hline Sé cómo actuar en una entrevista de selección para un empleo & 4,80 & 1,180 & 1,394 \\
\hline Sé cómo controlar mi ansiedad ante una entrevista de trabajo & 4,72 & 1,143 & 1,306 \\
\hline Tengo manejo para salir de situaciones incómodas asertivamente & 4,85 & 1,062 & 1,128 \\
\hline Sé elaborar un currículum para solicitar un puesto de trabajo & 5,28 & 0,836 & 0,698 \\
\hline Tengo facilidad para comunicarme con los demás & 5,25 & 1,055 & 1,113 \\
\hline Antes de entrar en este máster me sentía desorientado & 2,58 & 1,549 & 2,399 \\
\hline
\end{tabular}




\begin{tabular}{lllll}
\hline $\begin{array}{l}\text { Ahora sé perfectamente dónde puedo recibir la orientación que } \\
\text { necesito }\end{array}$ & 3,93 & 1,430 & 2,044 \\
\hline $\begin{array}{l}\text { Sé cómo planificar mi tiempo de estudio y de trabajo personal } \\
\text { Dispongo de suficiente información respecto a la estructura y a }\end{array}$ & 4,78 & 1,78 & 1,019 & 1,0387 \\
\hline $\begin{array}{l}\text { los servicios que ofrece la universidad } \\
\text { Grado de orientación y ayuda de mi familia más cercana }\end{array}$ & 3,86 & 1,831 & 3,352 \\
\hline Grado de orientación y ayuda de mi pareja & 3,42 & 1,940 & 3,762 \\
\hline Grado de orientación y ayuda de mis amistades & 3,11 & 1,546 & 2,389 \\
\hline Grado de orientación y ayuda de otros miembros de mi familia & 2,72 & 1,653 & 2,732 \\
\hline Grado de orientación y ayuda de profesorado universitario & 3,12 & 1,763 & 3,107 \\
\hline Grado de orientación y ayuda de la unidad de orientación de la US & 2,08 & 1,407 & 1,978 \\
\hline Grado de orientación y ayuda de otras personas & 2,60 & 2,044 & 4,179 \\
\hline
\end{tabular}

Fuente: elaboración propia.

A partir de los resultados expuestos en la tabla 6 , se observa que existe un conocimiento sobre la autoeficacia por parte de los encuestados, dato que sobresale entre los factores personales favorecedores de un proyecto profesional. Por el contrario, existe menos valoración de los factores externos relacionados con los sistemas de orientación por parte de la universidad, el apoyo por parte del profesorado y la ayuda de otras personas.

Algunos factores internos y externos son destacables en determinados grupos, por tanto, nos ocuparemos de exponer alguno de ellos.

1. Factores internos a las personas. La conciencia sobre la autoeficacia y la empleabilidad en los estudiantes de másteres puede explicar los recursos cognitivos $\mathrm{y}$ afectivos que tienen los estudiantes para enfrentarse a un proyecto profesional. A continuación se exponen algunos de ellos:

- El acceso a los puestos de trabajo nos puede permitir hablar de empleabilidad en los estudiantes de másteres. En los séniores se evidencia mayor conciencia sobre los puestos de trabajo a los que pueden acceder en relación con los júniores o recién titulados. Podemos observar diferencias entre los grupos $(p<0,05)$ que componen la categoría de respuesta acerca de la experiencia profesional júnior $(a)$ y sénior $(b)$, donde $b>a$, con relación a la toma de conciencia sobre los puestos a los que pueden acceder.

- Con relación a la facilidad de comunicación según la tipología profesional de acceso, encontramos diferencias $(p<0,05)$ entre los grupos que componen la categoría de respuesta experiencia profesional júnior (a) y sénior $(b)$, donde $b>a$ con relación a la facilidad para comunicarse con los demás. Los que revelan mayor consciencia de comunicación son los séniores y los recién titulados.

- El conocimiento sobre procedimientos de emprendedor puede ser un elemento predictor que exprese mayor o menor empleabilidad. Los resultados indican que existen diferencias entre las tipologías de estu- 
diantes. Los recién titulados conocen más sobre los procedimientos a seguir para emprender con una empresa propia (media $=4,9)$. Los júniores (media $=4,5)$ y los séniores (media $=4,4)$ tienen menor conocimiento sobre este procedimiento. Se evidencian diferencias significativas $(p<0,05)$ en relación con los titulados después de un año que todavía no han encontrado trabajo (media $=3,6$ ).

2. Factores externos. Las percepciones sobre los factores externos favorecen la transición académica en la medida en que permiten conocer los recursos de soporte que recibe el estudiante por parte de la universidad. De esta manera destacamos los siguientes factores:

- En los procesos de orientación (Antes de entrar en este máster me sentía desorientado) hay que destacar los bajos niveles de puntuación media con respecto a la media teórica. Aplicando las pruebas de comparación post oc, encontramos diferencias entre los grupos que componen la categoría de respuesta acerca de la experiencia profesional júnior $(a)$ y aquellos que acaban de terminar sus estudios $(c)$, donde $c>a(p<0,05)$. Es decir, los recién titulados perciben mayor orientación.

- Con relación al ítem Ahora séperfectamente dónde puedo recibir la orientación que necesito existen diferencias. Aplicando las pruebas de comparación de Tukey, Duncan y Scheffé, encontramos diferencias entre los grupos que componen la categoría de respuesta acerca de la experiencia profesional júnior $(a)$ y aquellos que acaban de terminar sus estudios $(c)$, donde $c>a(p<0,05)$. Los júniores son los que menor percepción sobre orientación han valorado (ver la tabla 7).

- Con relación a la información previa, en el ítem Me hubiese gustado contar con más ayuda e información antes de matricularme, se evidencia similitud entre los grupos que acaban de terminar los estudios (media $=3,10$ ), los júniores (media $=3,15)$ y los séniores (media $=3,35$ ). No así en los que han terminado el grado después de un año, pero que no cuentan con experiencia profesional (media $=4,35)$. Este último grupo es el menos satisfecho con la orientación y la información recibida antes de matricularse en el máster. En cuanto al ítem Conozco el lugar donde tengo que dirigirme para asesorarme sobre becas que me interesen y que me ayuden a continuar mis estudios, los menos informados son los titulados del grado después de un año y que no han trabajado $($ media $=2)$. Los más informados son los que se acaban de titular (media $=3,20)$.

El análisis ANOVA con el referente de la universidad en la que se realizan los estudios no muestra diferencias significativas en el nivel académico de satisfacción (con medias claramente superiores a la media teórica). 


\section{Las transiciones académicas según tipologías de acceso}

Los factores que facilitan las transiciones académicas pueden explicar la calidad educativa y los elementos que intervienen en la inclusión de estudios. De esta manera, la transición está condicionada, entre otras cuestiones, por factores cognitivos y afectivos.

La prueba de Duncan en el ítem sobre la exigencia de los estudios nos muestra diferencias significativas en su percepción $(p<0,05)$. El grupo que mayor exigencia encuentra en los estudios son los que los acaban de terminar (media $=5,5)$, y los que menos exigencia encuentran son los júniores (media $=3,6)$. Estas diferencias pueden deberse a la ausencia de experiencia profesional y a la menor estructura cognitiva asimilada. La transición analizada permite deducir la necesidad de que los estudiantes tengan experiencias laborales facilitadoras de transición académica de los estudios postgraduales (ver la tabla 7).

Otros ítems, tales como los relacionados con los problemas económicos, la inadecuación de la docencia, la percepción sobre la organización y sus dificultades y el poco apoyo familiar, evidencian la influencia en los procesos de transición, con puntuaciones claramente superiores a la media teórica en los grupos analizados.

Tabla 7. Motivos de abandono de los estudios de máster

\begin{tabular}{lccc}
\hline & Media & Ds & Vz \\
\hline Has pensado alguna vez en dejar los estudios de máster & 1,30 & 0,715 & 0,511 \\
\hline Exigencia alta / exigencia baja & 3,89 & 1,656 & 2,743 \\
\hline Estudios inadecuados / estudios adecuados & 4,39 & 2,241 & 5,024 \\
\hline Problema familiar o personal / ningún problema familiar o personal & 4,22 & 2,204 & 4,859 \\
\hline $\begin{array}{l}\text { Relación negativa con los compañeros / relación positiva con los } \\
\text { compañeros }\end{array}$ & 4,00 & 2,283 & 5,211 \\
\hline Problemas económicos / sin problemas económicos & 4,16 & 2,020 & 4,081 \\
\hline Contenido desinterés / contenido interés & 4,43 & 1,996 & 3,982 \\
\hline Inadecuación de la docencia / adecuación de la docencia & 3,83 & 1,739 & 3,024 \\
\hline Con dificultades de organización / sin dificultades de organización & 4,47 & 1,777 & 3,159 \\
\hline Poco apoyo familiar / bastante o mucho apoyo familiar & 4,47 & 2,113 & 4,466 \\
\hline $\begin{array}{l}\text { Relaciones con profesores influencia negativa / relaciones con pro- } \\
\text { fesores influencia positiva }\end{array}$ & 4,65 & 2,096 & 4,392 \\
\hline
\end{tabular}

Fuente: elaboración propia.

\section{Abandono de los estudios de máster}

Desde el análisis de la calidad, el abandono es un indicador importante a la hora de valorar la relación entre los objetivos del programa y las trayectorias profesionales. 
Entre los elementos que influyen para incidir sobre el abandono, con medias más altas, se mencionan los estudios inadecuados, los problemas familiares, los problemas económicos, los contenidos que generan desinterés, las dificultades en la organización, el poco apoyo familiar y la influencia negativa de las relaciones con los docentes.

\section{Conclusiones}

En general, podemos observar que existen diferentes percepciones según la experiencia profesional. La procedencia profesional es un elemento importante a la hora de valorar las trayectorias laborales y su relación con el cumplimiento de expectativas y factores de transición, debido a que las exigencias y las percepciones cambian con la experiencia y la edad. Es decir, se deben considerar las características sociodemográficas a la hora de analizar la transición académica. Dicha consideración es coincidente con otras investigaciones (Figuera y Torrado, 2013; AQU, 2003), en las que se consideran los aspectos sociodemográficos y características de las carreras como factores facilitadores de transición, tanto académica como profesional, En lo que respecta a la selección de estudios, se ven influenciados por las trayectorias profesionales. Para el grupo de júniores inciden aspectos relacionados con el cambio profesional, para el grupo de séniores intervienen factores relacionados con la especialización. Por tanto, las trayectorias profesionales y el momento influyen directamente en las motivaciones de la elección de estudios.

Respecto al proyecto académico y a los niveles de satisfacción, existen factores internos y externos que condicionan la percepción. Siguiendo a Lent (2004), existen factores sociocognitivos y de ajuste organizativo y académico que pueden explicarse desde las trayectorias profesionales. En el grupo encuestado, entre los aspectos que han destacado sobre los factores sociocognitivos, encontramos el acceso a los puestos de trabajo, la facilidad de comunicación y los procesos para constituir una empresa.

Entre los factores que dificultan la transición académica, se han destacado los problemas económicos, la inadecuación de la docencia, las dificultades de organización y el poco apoyo familiar. Los júniores son los que mayores problemas económicos encuentran, mientras que los séniores y los recién titulados perciben este elemento como facilitador de la transición. En cuanto a la práctica docente, se evidencia que se trata de un elemento facilitador para los júniores, pero no para los séniores ni los recién titulados. La percepción sobre la organización y sus dificultades es un tema inhibidor de la transición para los séniores y los recién titulados, mientras que para los júniores constituye un elemento facilitador. El grupo que recibe mayor apoyo familiar para los estudios es el de los júniores. Este no es el caso de los recién titulados y los séniores.

El análisis realizado nos permite concluir que existen diferencias en las percepciones según las tipologías de acceso y las características de la población. El cumplimiento de las expectativas y los factores facilitadores o inhibidores de la transición académica de los estudiantes de máster en Educación depen- 
den en gran medida de las trayectorias formativas y laborales realizadas por los postulantes.

\section{Referencias bibliográficas}

AQU (2003). Educació superior i treball a Catalunya. Barcelona: AQU.

Cabrera, A.; Burkum, K.R.; Lanasa, S.M. y Bibo, E.W. (2016). Pathways to a FourYear Degree: Determinants of Transfer and Degree Completion among Socioeconomically Disadvantaged Students. En A. Seidman (ed.). College student retention (pp. 35-51). Westport: Praeger Publishers.

CASAls, J. (1996). La inserción social y profesional de los jóvenes. Barcelona: Paidós.

CEUB (2015a). Normas y documentos. Recuperado de <www.ceub.edu.bo $>$.

- (2015b). Estadísticas en Educación Superior. Recuperado de <www.ceub.edu.bo>.

CraWford, K. y Harris, M. (2008). Differential predictors of persistence between community college adult and traditional aged students. Journal of Research and Practice, 32, 75-100.

Cox, A.; Jament, J. y TARRY, E. (2011). An investigation into parental expectations of Primary schooling and the support provided by schools to a single Keralite community in an English county. Procedia - Social and Behavioral Sciences, 15, 66-71. $<$ https://doi.org/10.1016/j.sbspro.2011.03.052>

Figuera, P. y ToRRADo, M. (2013). El contexto académico como factor diferenciador en la transición a la universidad. Revista Contrapontos, 12(1), 33-41.

Fike, D. y Fike, R. (2008). Predictors of First-Year Student Retention in the Community College. Community College Review, 36(2), 68-88.

Galindo, C. y Sheldon, S. (2012). School and home connections and children's: The mediating effects of family involvement. Early Childhood Research Quarterly, 27(1), 90-103. <https://doi.org/10.1016/j.ecresq.2011.05.004>

GlogowsKa, M.; Young, P. y Lockyer, L. (2007). Should I go o should I stay?: A study of factors influencing students' decisions early leaving. Active Learning in Higher Education, 8(1), 63-77.

Higher Education Funding Council for England (HEFCE) (2005). Sustainable Development in Higher Education. Recuperado de <https://dera.ioe.ac. uk/11513/1/05_28.pdf>.

IntXausti, N.; ETXEberRia, F. y JoAristi, L. (2014). ¿Coinciden las expectativas de la familia y del profesorado acerca del alumnado de origen inmigrante? Relieve, 20(1), 1-21. $<$ https://doi.org/10.7203/relieve.20.1.3804>

IsHITANI, T.T. (2008). How to explore timing of intervention for student at risk of departure. En T.T. IsHITANI (ed.). Alternative perspectives in institutional planning: New directions for institutional research (pp. 25-36). San Francisco: Jossey-Bass.

Justiniano, M. (2007). La inserción laboral de los profesionales graduados en Ciencias de la Educación. Tesis doctoral. Barcelona: Universidad de Barcelona.

Karp, M.M. (2011). How non-academic supports work: Four mechanisms for improving student outcomes. CCRC, Columbia University.

Krause, K. y Coates, H. (2008). Students' engagement in first-year university. Assessment and Evaluation in Higher Education, 33(5), 1-15.

LazARus, R.S. y Folkman, S. (1996). Estrés y procesos cognitivos. Barcelona: Martínez Roca. 
Lent, R.W. (2004). Toward a Unifying Theoretical and Practical Perspective on WellBeing and Psychosocial Adjustment. Journal of Counseling Psychology, 51(4), 482509.

Lent, R.W.; Taveira, M.D.; Sheu, H. y Singley, D. (2009). Social cognitive predictors of academic adjustment and life satisfaction in portuguese college students: A longitudinal analysis. Journal of Vocational Behavior, 74, 190-198.

«Ley de la Educación N. 70 "Avelino Siñani - Elizardo Pérez"». Gaceta Oficial del Estado Plurinacional de Bolivia (10 de junio de 2012).

LILLIS, M.P. (2012). Faculty emotional intelligence and student-faculty interactions: Implications for student retention. Journal of College Student Retention: Research, Theory \& Practice, 13(2), 155-178.

Lotкowski, V.; Robbins, S. y Noeth, R. (2004). The role of academic an non-academic factors in improving college retention. ACT Policy Report. Iowa: ACT, Inc.

Nonis, S.A. y Hudson, G.I. (2010). Performance of college students: Impact of study time and study habits. Journal of Education for Business, 85(4), 229-238.

OJeda, L.; Navarro, R. y Flores, L. (2011). Social cognitive predictors of mexican collage students academic and life satisfaction. Journal of Counseling Psychology, 58, 61-71.

Pascarella, E. y Terenzini, P.T. (2005). How college affects students. Vol. 2. A third decade of research. San Francisco: Jossey-Bass.

Perna, L. y Titus, M. (2005). The relationship between parental involvement as social capital and college enrollment: An examination of racial/ethnic group differences. Journal of Higher Education, 76(5), 485-518.

Rivera, M. y Milicic, N. (2006). Alianza familia-escuela: Percepciones, creencias, expectativas y aspiraciones. Pybie, 15(1), 1-15.

Scott-Clayton, J. (2011). The shapeless river: Does a lack of structure inhibit students' progress at community colleges? CCRC working paper, 25.

Strydom, J.F. y Mentz, M. (2010). Focusing the student experience on success through student engagement. Pretoria: Council on Higher Education.

Stull, J. (2013). Family socioeconomic status, parent expectations, and a child's achievement. Research in Education, 90, 53-67. $<$ https://doi.org/10.7227/RIE.90.1.4>

Tinto, V. (1988). Stages of Student Departure: Reflections on the Longitudinal Character of Student Leaving. The Journal of Higher Education, 59(4), 438-455. $<$ https://doi.org/10.2307/1981920>

Torrado, M. (2012). El fenómeno del abandono en la Universidad de Barcelona: El caso de ciencias experimentales. Tesis doctoral. Universidad de Barcelona.

VRoom, V.H. (1964). Work and motivation. San Francisco, CA: Jossey-Bass.

Weinberg, L. (2009). Parents' educational expectations for their young children: SES, racelethnicity and school feedback. Tesis doctoral. The Florida State University.

Yorke, M. y LONGDen. B. (2008). The first-year experience of higher education in the $U K$. York, UK: The Higher Education Academy. 